\title{
Hypermethylation of the gene promoter and enhancer region can regulate Fas expression and sensitivity in colon carcinoma
}

\author{
I Petak ${ }^{1,2}$, RP Danam ${ }^{1,3}$, DM Tillman², R Vernes ${ }^{2}$, SR Howell ${ }^{3}$, \\ L Berczi ${ }^{4}$, L Kopper ${ }^{4}$, TP Brent ${ }^{3,5}$ and JA Houghton ${ }^{\star 2,5}$ \\ ${ }^{1}$ Both authors contributed equally to this work \\ 2 Division of Molecular Therapeutics, Department of Hematology-Oncology, \\ Memphis, TN, USA \\ ${ }^{3}$ Department of Molecular Pharmacology, St. Jude Children's Research \\ Hospital, 332 North Lauderdale, Memphis, TN 38105, USA \\ ${ }^{4}$ Semmelweis University of Medicine, 1st Institute of Pathology and \\ Experimental Cancer Research, Budapest 1085, Hungary \\ ${ }^{5}$ Both authors contributed equally to the senior authorship of this manuscript \\ * Corresponding author: JA Houghton, Division of Molecular Therapeutics, \\ Department of Hematology-Oncology, St. Jude Children's Research Hospital, \\ 332 North Lauderdale, Memphis, TN 38105, USA. Tel: +1 901495 3456; \\ Fax: +1 901523 2622; E-mail: janet.houghton@stjude.org
}

Received 4.12.01; revised 1.8.02; accepted 2.8.02

Edited by Carol Thiele

\section{Abstract}

Expression of the cell surface receptor Fas is frequently lost or decreased during tumor progression in human colon carcinomas. The methylation status of a $583 \mathrm{bp} \mathrm{CpG-rich}$ region within the Fas promoter $(-575$ to +8$)$ containing 28 CpG sites was determined in human colon carcinoma cell lines. In Caco2 (no Fas expression), 82-93\% of CpG sites were methylated, whereas none were methylated in $\mathrm{GC}_{3} / \mathrm{c} 1$ (high Fas expression). In RKO (intermediate level of Fas), a single CpG site, located at -548 , was $100 \%$ methylated. The inhibitor of methylation, 5-aza-2'-deoxycytidine (5-azadC), upregulated Fas expression in four of eight cell lines, and sensitized RKO cells to recombinant FasL-induced apoptosis. The p53binding region in the first intron of the Fas gene was partially methylated in Caco2, and 5-azadC potentiated Ad-wtp53induced upregulation of Fas expression. Methylation-specific PCR of the first intron detected partial methylation in four out of 10 colon carcinoma tumor samples in vivo. The data suggest that DNA hypermethylation is one mechanism that contributes to the downregulation of Fas expression and subsequent loss of sensitivity to Fas-induced apoptosis in colon carcinoma cells.

Cell Death and Differentiation (2003) 10, 211-217. doi:10.1038/ sj.cdd. 4401132

Keywords: Fas; hypermethylation; p53; apoptosis; colon carcinoma

Abbreviations: 5-azadC, 5-aza-2'-deoxycytidine; FUra, 5-fluorouracil; IFN- $\gamma$, interferon- $\gamma$

\section{Introduction}

The cell surface receptor Fas (APO-1; CD95) is a member of the family of death receptors that induce apoptosis in sensitive cells upon binding to their specific death ligands. Fas and its ligand (FasL) are known regulators of apoptosis in cells of the immune system. ${ }^{1}$ Analyses of tissues from mice have demonstrated expression of Fas in tissues largely characterized by high rates of cell turnover and apoptotic cell death, including epithelial tissues. ${ }^{2}$ Fas is highly expressed in normal human colonic epithelial cells and its expression is progressively decreased during tumor progression from normal epithelium to adenocarcinoma in $\approx 50 \%$ of the cases. ${ }^{3,4}$ Several reports have demonstrated activation of the Fas signaling pathway in the antitumor activity of 5-fluorouracil (FUra), the most important chemotherapeutic agent used in the therapy of colon carcinomas ${ }^{5-8}$ We have demonstrated previously that sensitivity to Fas-mediated apoptosis correlated with the level of Fas expressed in human colon carcinoma cell lines. ${ }^{9}$ No mutations were detected in the Fas CDNA, and no gross gene rearrangements or frequent allelic loss of the Fas gene have been reported to date. ${ }^{10}$ Further, upregulation of Fas expression using the cytokine IFN- $\gamma$ sensitized cells to Fas-mediated and FUra/LV-induced cytotoxicity in a Fas-dependent manner. ${ }^{6}$ However, the mechanism of loss of Fas expression and decreased sensitivity to Fas-mediated apoptosis in colon carcinomas remains to be determined. The current study examined whether Fas expression was downregulated by DNA hypermethylation, and whether inhibition of DNA methylation by 5azadC could upregulate Fas expression and sensitize cells to Fas-mediated apoptosis.

Methylation is the main epigenetic modification in humans, and changes in patterns of DNA methylation play an important role in tumorigenesis. There is increasing evidence that de novo methylation of promoter-associated $\mathrm{CpG}$ islands is involved in the transcriptional silencing of several genes and contributes to the alteration of gene expression in cancer. These genes include not only classical tumor suppressor genes involved in cell cycle regulation or DNA repair, but also genes important in apoptosis such as caspase- $8^{11}$ and Apaf- $1 .{ }^{12}$

The human Fas gene contains a $650 \mathrm{bp} \mathrm{CpG}$ island spanning the $5^{\prime}$ flanking region of the gene, suggesting that $\mathrm{CpG}$ methylation could be responsible for downregulating Fas expression. ${ }^{13}$ The first intron, which contains a p53 responsive element, is also a region demonstrating high density of CpG sites. ${ }^{14}$ This study determined that DNA methylation contributes to the downregulation of Fas expression in $50 \%$ of colon carcinoma cell lines examined. Inhibition of methylation by 5 -azadC increased Fas expression, restoring sensitivity to Fas-mediated apoptosis and potentiated p53-induced upregulation of Fas expression. 

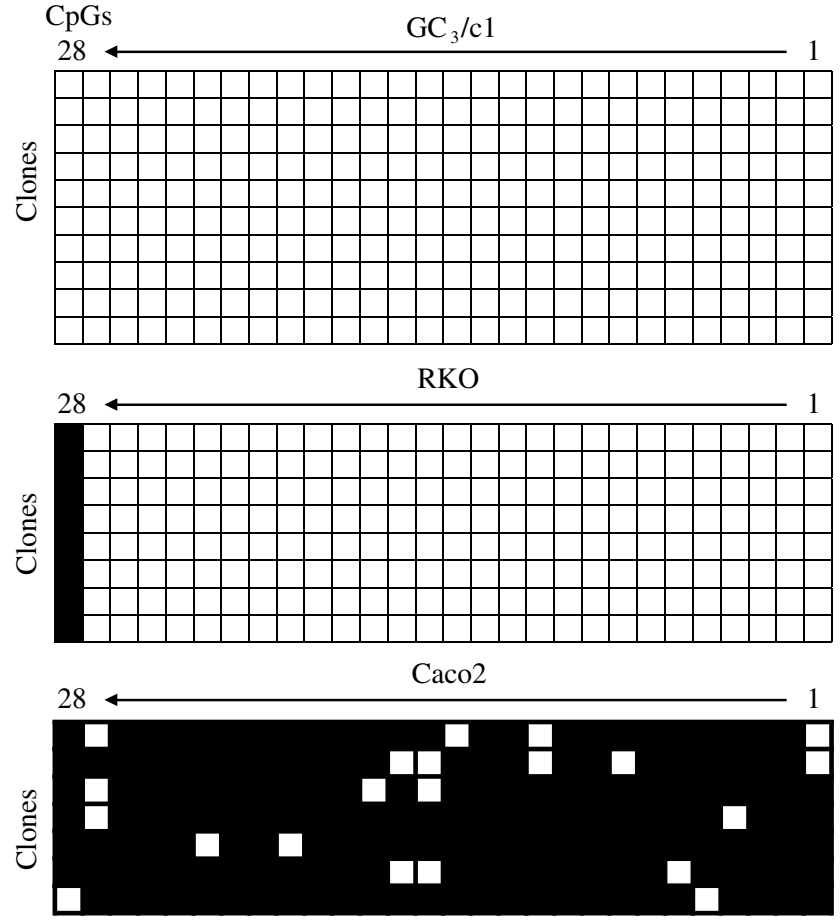

Figure 1 Bisulfite genomic sequencing of the Fas promoter in human colon carcinoma cell lines. Methylation patterns of individual plasmid clones of PCR products derived from $\mathrm{GC}_{3} / \mathrm{c} 1$, RKO and Caco2 cells were determined as described in the Materials and Methods section. Columns labeled from 1 to 28 represent the $\mathrm{CpG}$ sites and the rows demonstrate the sequencing of individual clones: ( $\boldsymbol{\square}$ ) methylated cytosine; $(\square)$ unmethylated cytosine

\section{Results}

\section{CpG island methylation}

The methylation status of $28 \mathrm{CpG}$ sites in the promoter region of the Fas gene was initially examined in $\mathrm{GC}_{3} / \mathrm{c} 1, \mathrm{RKO}$ and Caco2 human colon carcinoma cell lines using bisulfite genomic sequencing (Figure 1). Bisulfite modification of genomic DNA converts unmethylated deoxycytidine into uracil (read as thymidine) but methylated deoxycytidine remains unmodified. A $583 \mathrm{bp}$ region within the $5^{\prime}$ promoter ( -575 to +8 nucleotide relative to the transcriptional start site) containing the $\mathrm{CpG}$ island was PCR amplified from the bisulfite-treated DNA, and the PCR fragments were cloned and sequenced, thereby allowing identification of the methylation status of each of the 28 specific CpG sites in the Fas gene. In $\mathrm{GC}_{3} / \mathrm{c} 1$ cells with high levels of Fas expression, no $\mathrm{CpG}$ sites were methylated in the 10 clones analyzed. In RKO cells with moderate levels of Fas expression, a single CpG site located at position -548 was methylated in all eight clones. In Caco2 cells, which do not express Fas, 14 CpG sites were $100 \%$ methylated, at least 23 sites out of 28 were $82 \%$ methylated in all seven clones (Figure 1).

\section{DNA methylation and Fas expression}

To provide evidence for a possible role of DNA methylation in the regulation of Fas expression, cell surface Fas levels were analyzed by flow cytometry both before and after treatment with the demethylating agent 5-azadC $(1 \mu \mathrm{M})$ for $72 \mathrm{~h}$ (Figure 2a). The calibration of red fluorescence intensity of PElabeled anti-Fas monoclonal antibodies with QuantiBright beads, covered with a known number of PE molecules enabled quantitative comparison of Fas expression among the different samples. Further, assuming a $P E: A B$ ratio of 1 , the number of PE molecules detected on the cell surface closely correlated with the actual number of Fas molecules. Fas expression was highest in $\mathrm{GC}_{3} / \mathrm{c} 1$ cells $\left(6.68 \times 10^{4} \mathrm{PE}\right.$ molecules/cell) and was not changed significantly by 5 -azadC treatment $(P<0.1$; Figure 2a). The level of Fas was lower in RKO (2.66 $\times 10^{4} \mathrm{PE} /$ cell $)$ and was significantly upregulated by three-fold to $8.67 \times 10^{4} \mathrm{PE} /$ cell in the presence of 5 -azadC $(P<0.001)$. Constitutive Fas expression was not detectable in Caco 2 cells ( $P<0.4$ compared to isotype AB control), but after 5-azadC treatment Fas expression became detectable $\left(0.12 \times 10^{4} \mathrm{PE} / \mathrm{cell} ; P<0.04\right)$. These results indicate a close correlation between the methylation status of the Fas gene, the level of cell surface Fas expressed, and the suppression of Fas expression by methylation in RKO and Caco2 cells. Subsequently, the correlation between Fas expression and sensitivity to Fas-mediated apoptosis of these colon carcinoma cell lines was determined (Figure $2 b$ ). Both $\mathrm{CH}-11$ (200 ng/ $\mathrm{ml})$ and recombinant human $\mathrm{FasL}(50 \mathrm{ng} / \mathrm{ml})$ induced a $50 \%$ increase in apoptosis in $\mathrm{GC}_{3} / \mathrm{c} 1$ cells following a $72 \mathrm{~h}$ exposure. The sensitivity of $\mathrm{GC}_{3} / \mathrm{c} 1$ (expressing high levels of Fas) to Fas-induced apoptosis did not change after treatment with 5-azadC. In contrast, RKO cells were resistant to both $\mathrm{CH}$-11- and FasL-induced apoptosis in the absence of 5 -azadC, but were sensitized after 5-azadC treatment in response to $\mathrm{CH}-11$ (25\% apoptosis) or FasL (40\% apoptosis, $P<0.01)$. Caco2 cells were not sensitive to either $\mathrm{CH}-11$ or FasL and were not sensitized in the presence of 5-azadC.

To confirm that hypermethylation of the CpG site at -548 decreased after 5-azadC treatment, RKO cells were treated with 5 -azadC $(72 \mathrm{~h}, 1 \mu \mathrm{M})$ and the methylation status of the Fas promoter was analyzed by bisulfite genomic sequencing in both the control and treated samples (Figure 3). In the control sample, the CpG -548 was $71 \%$ methylated (five out of seven clones) and only $29 \%$ (two out of seven clones) methylated in the treated sample. Data suggest that demethylation of this $\mathrm{CpG}$ can have a role in the 5-azadCinduced upregulation of Fas in this cell line.

\section{Influence of wtp53}

Transcriptional regulation of Fas by wtp53 has been reported in these cell lines. ${ }^{15}$ We therefore examined whether hypermethylation of the Fas gene would affect the p53dependent transcriptional activation of Fas expression. A putative p53 enhancer region exists in the first intron of the Fas gene, ${ }^{14}$ and contains $23 \mathrm{CpG}$ sites. We therefore determined the methylation status of the $p 53$ enhancer region by methylation-sensitive PCR (Figure 4a). There was no detectable methylation of the enhancer region in $\mathrm{GC}_{3} / \mathrm{c} 1$ or RKO cell lines (Figure 4a). Partial methylation of this sequence observed in Caco2 cells disappeared after the treatment with 5-azadC (Figure 4b). To ascertain whether inhibition of DNA hypermethylation by 5 -azadC increased the upregulation of Fas expression by wtp53, Caco2 cells were 

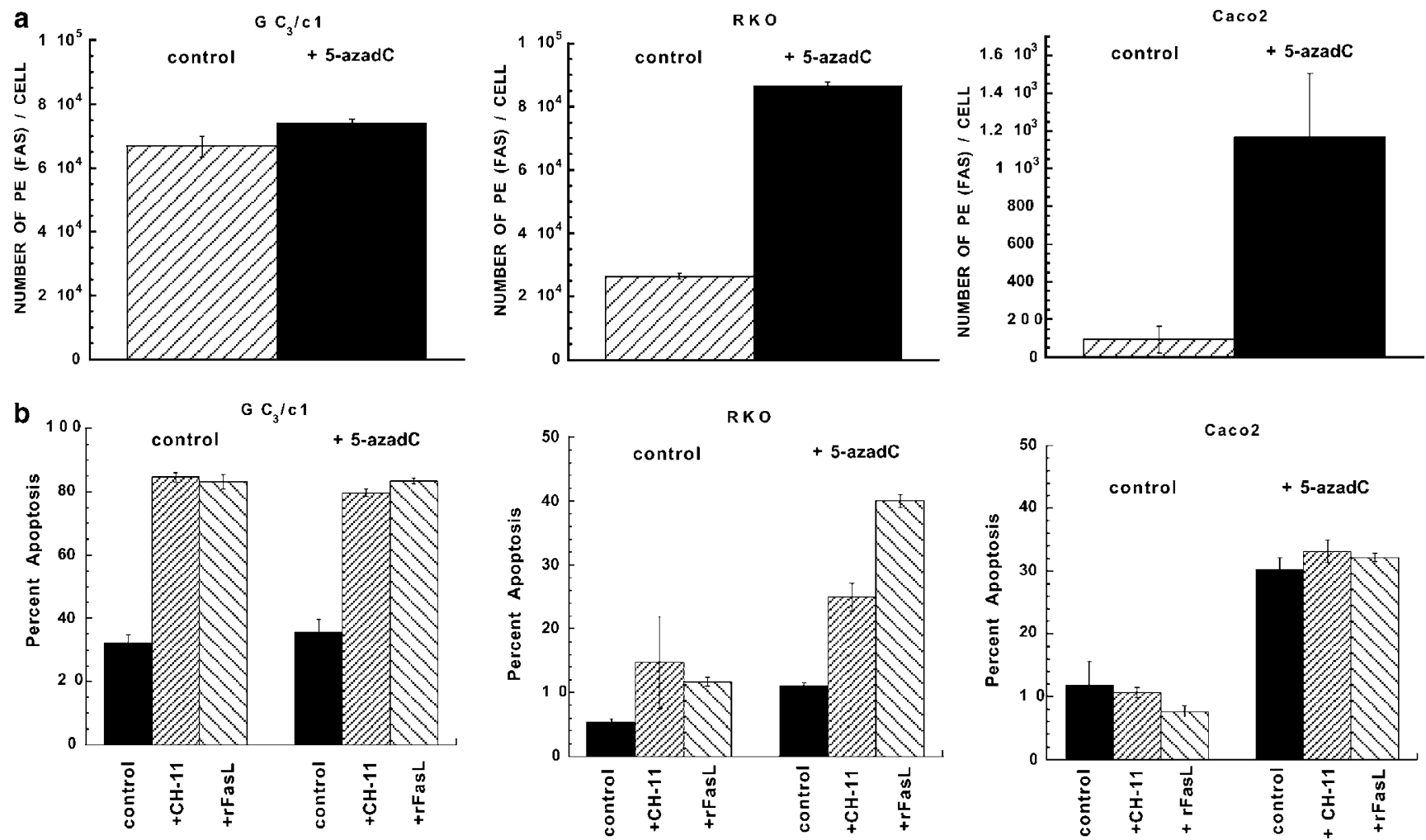

Figure 2 (a) Expression of cell surface Fas. (b) FACS analysis of apoptosis induced in cells in the absence or presence of 5 -azadC $(1 \mu \mathrm{M}), \mathrm{CH}-11(200 \mathrm{ng} / \mathrm{ml})$ or recombinant FasL $(50 \mathrm{ng} / \mathrm{ml})$ for $72 \mathrm{~h}$. Data represent the mean \pm S.D. of four determinations per point
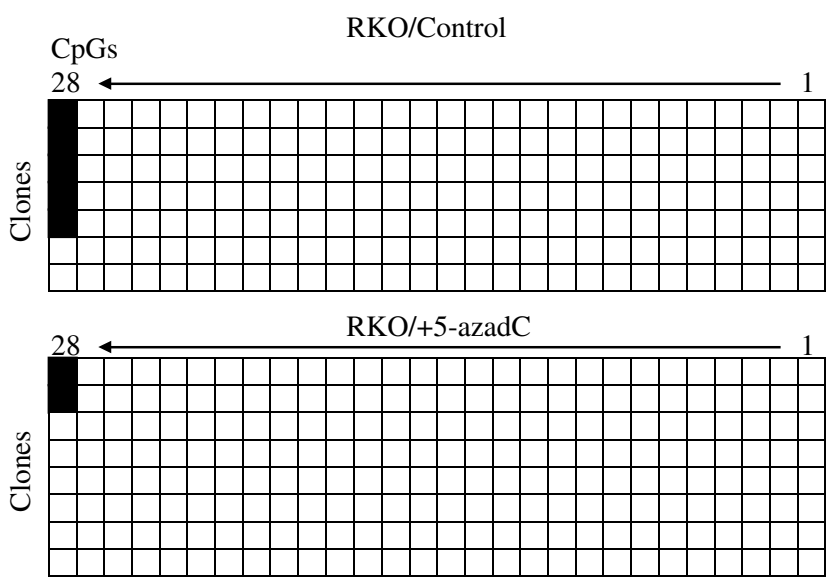

Figure 3 Bisulfite genomic sequencing of the Fas promoter in the RKO cell line before (control) and after treatment with 5 -azadC $(72 \mathrm{~h} ; 1 \mu \mathrm{M} ;+5$-azadC) Methylation patterns of individual plasmid clones were determined as described in the Materials and Methods section. Columns labeled from 1 to 28 represent the $\mathrm{CpG}$ sites and the rows demonstrate the sequencing of individual clones: ( $\mathbf{\square}$ methylated cytosine; $(\square)$ unmethylated cytosine

transduced with Ad-wtp53 and treated with 5-azadC $(1 \mu \mathrm{M}$, $72 \mathrm{~h}$; Figure $5 \mathrm{a})$. In the absence of 5 -azadC, Fas was undetectable in cells transduced with the empty vector. However, in cells transduced with Ad-wtp53, Fas was

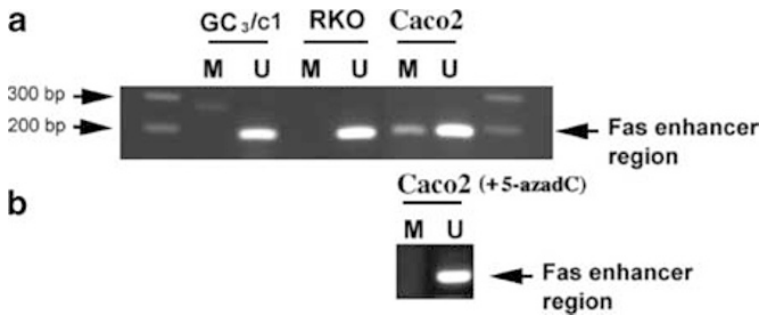

Figure 4 Methylation analysis of the Fas enhancer region in colon carcinoma cell lines. (a) Methylation status of the $\mathrm{CpG}$-rich Fas enhancer region (first intron containing the p53-binding region) was analyzed in $\mathrm{GC}_{3} / \mathrm{c} 1$, RKO and Caco2 cells using methylation-sensitive PCR. (b) Methylation status of the same region in Caco2 cells after treatment with 5-azadC $(1 \mu \mathrm{M})$ for $72 \mathrm{~h}$. Lane M: amplified product with primers recognizing the methylated Fas enhancer sequence. Lane $\mathrm{U}$ : amplified product with primers recognizing the unmethylated Fas enhancer sequence

moderately upregulated up to $4.6 \times 10^{3} \mathrm{PE} / \mathrm{cell}$ at an moi of 100. 5-azadC alone upregulated Fas to $2.7 \times 10^{3} \mathrm{PE} /$ cell. The combination of Ad-wtp53-transduction and 5-azadC treatment induced more than six-fold increase in Fas expression compared to either treatment alone. The combination of $\mathrm{Ad}$ wtp53 (moi 100) and treatment with 5-azadC (1 $\mu \mathrm{M})$ for $48 \mathrm{~h}$ (but neither alone) demonstrated slight enhancement of the sensitivity of Caco2 cells to $\mathrm{CH}-11(200 \mathrm{ng} / \mathrm{ml}, 24 \mathrm{~h}$; Figure $5 b)$. The protein synthesis inhibitor cycloheximide ( $\mathrm{CHX}$, 


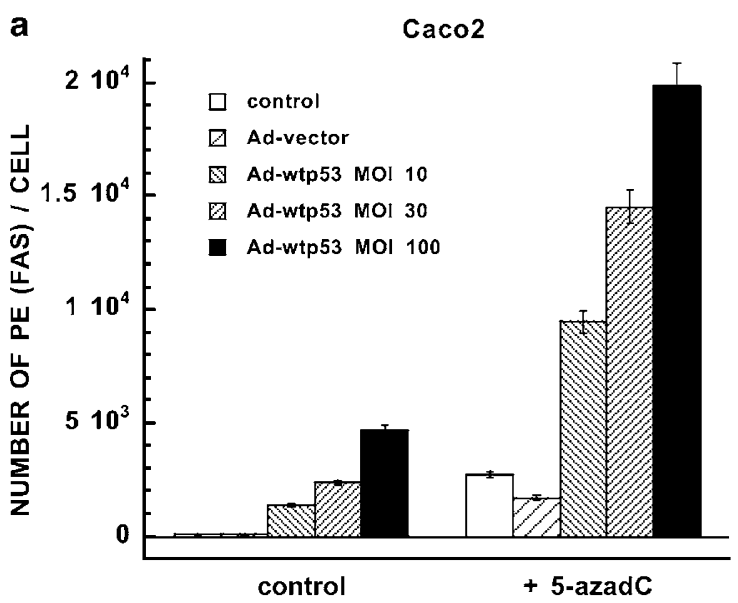

b

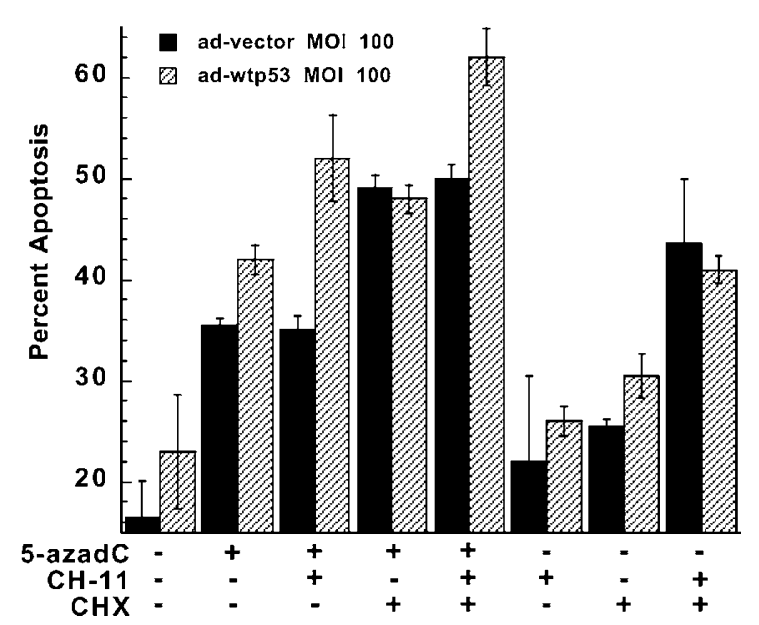

Figure 5 (a) Fas expression was analyzed by FACS in Caco2 cells transduced with Ad-wtp53 (moi 10-100) alone or following treatment with 5-azadC (1 $\mu \mathrm{M})$ for $72 \mathrm{~h}$. Data are the mean \pm S.D. of four determinations. (b) FACS analysis of apoptosis induced in Caco2 cells transduced with Ad-wtp53 (moi 100) or Ad-VC (moi 100) alone or in the presence 5 -azadC $(1 \mu \mathrm{M})$ for $48 \mathrm{~h}$ and subsequently treated with $\mathrm{CH}-11(200 \mathrm{ng} / \mathrm{ml})$ for $24 \mathrm{~h}$ in the presence or absence of cycloheximide $(\mathrm{CHX} ; 10 \mu \mathrm{M})$. Data are the mean \pm S.D. of two determinations

$10 \mu \mathrm{M})$ alone sensitized adenovirus-transduced Caco2 cells to $\mathrm{CH}-11$. This indicates that not only the level of Fas receptor but also other downstream molecular factors regulate Fas sensitivity. Thus, the combination of Ad-wtp53 and 5-azadC further sensitized Caco2 cells to $\mathrm{CH}-11$ in the presence of cycloheximide (Figure 5b).

\section{Effects of 5-azadC on transcription}

To determine whether 5-azadC increased Fas expression at the transcriptional level, levels of Fas mRNA were analyzed in RKO and Caco2 cells and in six additional colon carcinoma cell lines before and after treatment with 5-azadC $(1 \mu \mathrm{M})$ for $72 \mathrm{~h}$ (Figure 6). Fas mRNA was upregulated in SW480, RKO, HCT8 and Caco2 cell lines. In Caco2 cells, Fas mRNA was detectable only after 5-azadC treatment. There was no change in Fas expression in $\mathrm{GC}_{3} / \mathrm{c} 1, \mathrm{HT} 29, \mathrm{VRC}_{5} / \mathrm{c} 1$ or HCT116 after 5-azadC treatment. Thus, $50 \%$ of the colon

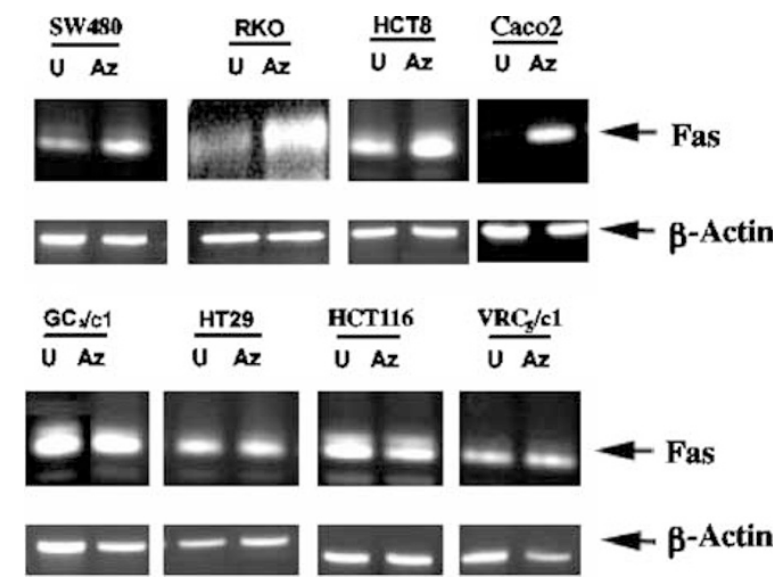

Figure 6 Upregulation of Fas Expression by 5-azadC in eight human colon carcinoma cell lines. Levels of Fas mRNA were determined by RT-PCR in untreated $(\mathrm{U})$ and 5 -azadC-treated cells (Az; $(1 \mu \mathrm{M}$ for $72 \mathrm{~h})$. Expression of $\beta$ actin served as the internal control

carcinoma cell lines examined demonstrated methylationdependent regulation of Fas expression. Further, HT29, HCT8, HCT116, VRC $5 / c 1$ and SW480 were not sensitized to $\mathrm{CH}-11$ or FasL following pretreatment with 5-azadC (data not shown), indicating that Fas upregulation by 5 -azadC is necessary but not sufficient to sensitize cells to Fas-mediated apoptosis.

\section{DNA methylation and Fas expression in vivo}

To explore DNA hypermethylation and Fas expression in primary colon carcinoma samples in vivo, paraffin-embedded archived tumor material derived from 10 independent cases were analyzed. Immunohistochemical staining of tissue sections revealed strong membrane expression of Fas in normal colon epithelium and absent or very low expression in colon carcinoma cells in all cases (Figures 7 and 8). Tissue sections from the same paraffin blocks were also used to extract genomic DNA, which was subsequently bisulfite treated. Methylation-specific PCR of the p53 enhancer region detected the presence of unmethylated DNA in all tumor samples. In four out of 10 tumor samples, methylation was detected, the signal being strong in tumors \#8 and \#9 (Figure 8). These results indicate that methylation of the Fas enhancer region occurs in vivo and may contribute to the downregulation of Fas expression in a subset of colon carcinomas.

\section{Discussion}

This report demonstrates correlation between suppressed Fas expression and promoter hypermethylation in human colon carcinoma cell lines. We examined $583 \mathrm{bp}$ of the approximately $650 \mathrm{bp} \mathrm{GC-rich} 5^{\prime}$ regulatory region of the Fas gene and determined the methylation status of the 28 $\mathrm{CpG}$ sites in this region. The results provide evidence that DNA hypermethylation is one mechanism by which Fas expression can be downregulated in colon carcinoma cells. Consistent with this notion, inhibition of methylation with 5- 


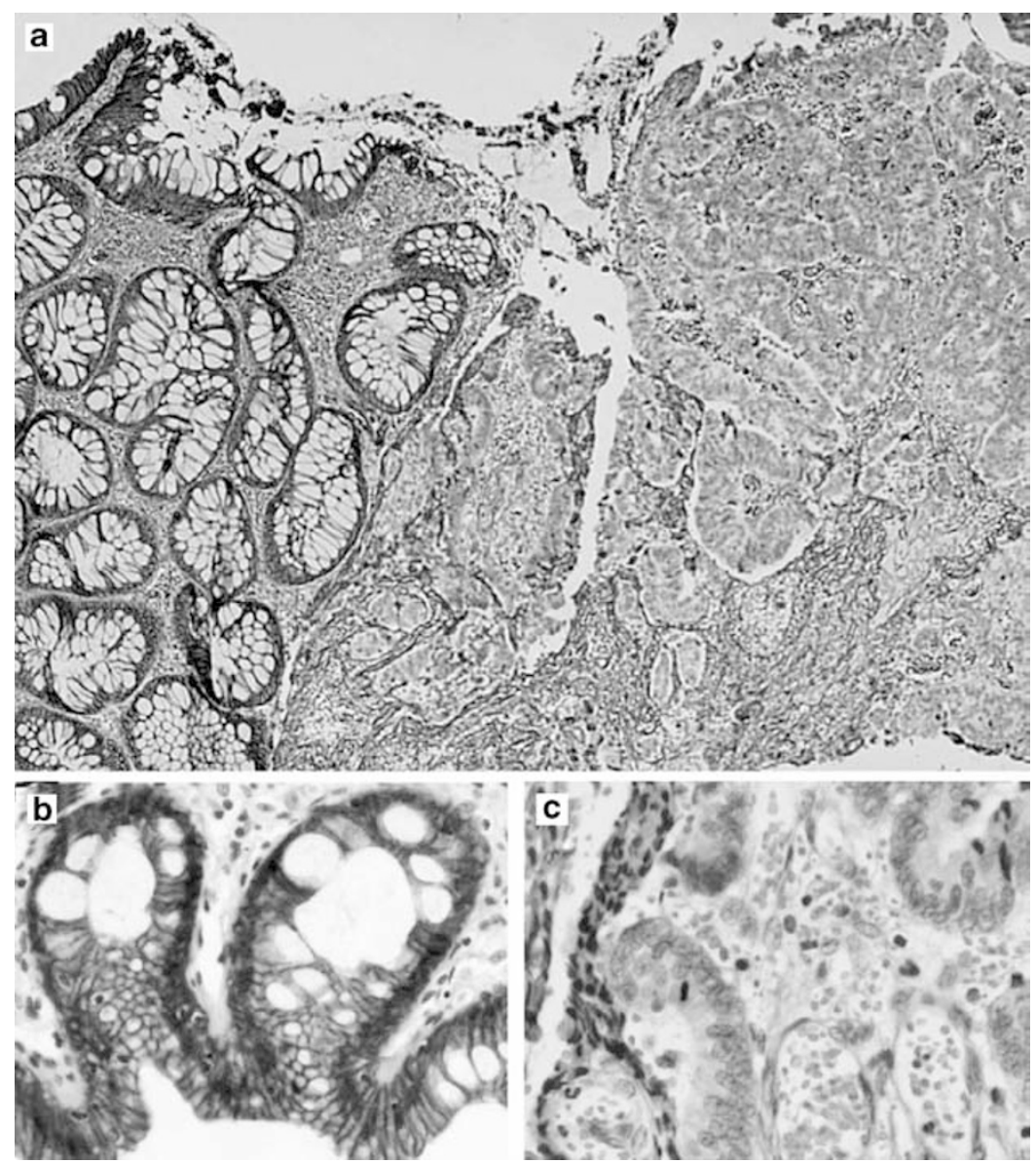

Figure 7 Immunohistochemical detection of Fas expression: (a) Low-power magnification $(\times 100)$ of neoplastic colonic epithelium (Fas negative) and adjacent normal colon (Fas positive). (b) high power $(\times 260)$ magnification of normal colon. (c) high-power $(\times 200)$ magnification of tumor tissue. Sections were counterstained with hematoxylin

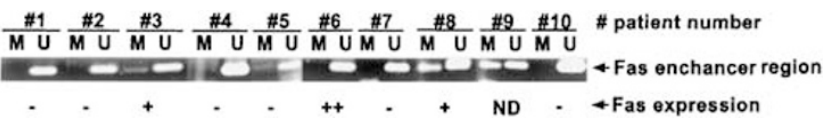

Figure 8 Methylation analysis of the Fas enhancer region in colon carcinoma specimens. Methylation status of the Fas enhancer region was analyzed using methylation specific PCR in 10 specimens of colon carcinoma. Lane M: amplified product with primers recognizing the methylated Fas enhancer sequence. Lane $\mathrm{U}$ : amplified product with primers recognizing unmethylated Fas enhancer sequence. The results of immunohistochemical studies for Fas expression were assessed by scoring the intensity of immunoreactivity relative to control staining using a scale: '-', negative; '+', weak; '++++', strong

azadC treatment in Caco2 cells resulted in the induction of Fas expression. However, these cells were not sensitized to FasL or to $\mathrm{CH}-11$. This may be because of the relatively low absolute number of Fas receptors on the cell surface after 5azadC treatment compared to the high Fas levels detected in sensitive cells. Since the Fas promoter in Caco2 cells is heavily methylated, demethylation by 5 -azadC alone may be inadequate to upregulate the level of Fas expression to threshold levels. However, Caco2 cells transduced with Adwtp53 were slightly sensitized to $\mathrm{CH}-11$ in the presence of 5azadC.
Our study also indicates that there are additional mechanisms responsible for the downregulation of Fas in certain cell lines. For example, 5-azadC did not upregulate Fas expression in $\mathrm{HT}_{2} 9, \mathrm{VRC}_{5} / \mathrm{c} 1$ or $\mathrm{HCT} 116$ cells, although these cell lines express intermediate levels of Fas and are relatively resistant to Fas-mediated apoptosis. ${ }^{9}$ The promoter region of Fas has binding sites for several transcription factors including p53, c-myb, NF- $\kappa \mathrm{B}, \mathrm{Sp}-1$ and IFN- $\gamma$ response elements such as GAS. ${ }^{13} \mathrm{P} 53$ and IFN- $\gamma$ are known regulators of Fas expression. P53 is inactivated by mutation or allelic loss in $75 \%$ of colorectal cancers, and hence may contribute to decreased Fas expression in this tumor type. Our results show that hypermethylation of the Fas gene inhibited the upregulation of Fas by wtp53; hence DNA methylation can suppress Fas expression despite the presence of a wtp53 gene.

One CpG site only (position -548) was methylated in RKO cells, and this site was frequently methylated in other cell lines of various origins (data not shown). In RKO, 5-azadC upregulated Fas expression several fold and sensitized the cells to FasL-induced apoptosis. Previously, Butler et al. ${ }^{16}$ used methylation-sensitive restriction enzymes and Southern blotting to evaluate the methylation status of $9 \mathrm{CpG}$ sites in the Fas promoter. These were determined to be unmethylated in 
samples from colorectal carcinomas and adenomas, suggesting that DNA methylation could not account for the frequent loss of expression of the Fas gene in colorectal carcinomas. However, a considerable area of the Fas promoter was not investigated, including the CpG site -548. In addition, a higher density region of $\mathrm{CpG}$ s exists in the enhancer region of the first intron, which has not been analyzed previously. Here, we demonstrated that partial methylation of this region occurs in a subset of colon carcinomas.

The antitumor activity of FUra has a Fas-dependent component in colon carcinoma cell lines ${ }^{6}$ and other cell types, ${ }^{8}$ and is influenced by the level of Fas expressed. Upregulation of Fas by the cytokine IFN- $\gamma$ sensitized colon carcinoma cells to FUra/LV. ${ }^{6}$ Our results suggest that inhibitors of methylation also have the potential to upregulate Fas expression independent of the p53 status in colon carcinomas and sensitize the cells to cytotoxic agents, in particular FUra/LV, that utilize the Fas signaling pathway to induce apoptosis and cytotoxicity.

\section{Materials and Methods}

\section{Cell lines}

The HT29, HCT8 and HCT116 human colon carcinoma cell lines were obtained from ATCC. $\mathrm{GC}_{3} / \mathrm{c} 1$ and $\mathrm{VRC}_{5} / \mathrm{c} 1$ were established as previously reported, ${ }^{9}$ and RKO was obtained from Dr. Michael Kastan, St. Jude Children's Research Hospital. Cells were maintained in the presence of folate-free RPMI 1640 medium containing 10\% dFBS and $80 \mathrm{nM}(6 \mathrm{RS}) 5$ methyltetrahydrofolate.

\section{Tissue samples}

Ten colon carcinoma tissue samples were obtained from the tissue bank of the Ist Institute of Pathology and Experimental Cancer Research, Semmelweis University, Budapest, Hungary. The material had been fixed in buffered formalin and embedded into paraffin.

\section{Genomic sequencing of bisulfite-modified DNA}

The selected 583 bp region extending between bp -575 and +8 was PCR amplified from bisulfite-converted genomic DNA extracted from $\mathrm{GC}_{3} / \mathrm{c1}$, RKO, Caco2 colon carcinoma cell lines and RKO cells treated with 5azadC $(72 \mathrm{~h} ; 1 \mu \mathrm{M}) .{ }^{17}$ The following primers were used: $5^{\prime}$ GGTTTTTGTATTTAGGTAGGATTTTTG (F) and 5'-CCCAACATAATTATTAAACAATCCTC $(\mathrm{R})$, corresponding to nucleotides -575 to -549 and +8 to -18 , respectively. The PCR product was ligated into the $P C R$ 2.1-TOPO vector and transformed into Top $10 \mathrm{~F}^{\prime}$ cells using the TOPO TA Cloning kit (Invitrogen, Carlsbad, CA, USA). Clones containing the correct insert were identified by EcoR 1 restriction enzyme digestion. Plasmid DNAs were isolated from multiple clones from each colon carcinoma cell line (Qiagen, Chatsworth, CA, USA). DNA sequencing was performed by an automated $\mathrm{ABI}$ Prism Big Dye Terminator Cycle Sequencing System (Hartwell Center for Bioinformatics \& Biotechnology, St. Jude Children's Research Hospital). The known genomic DNA sequence was compared to the sequence derived from the bisulfite-modified DNA for each clone, and the methylation status of each $\mathrm{CpG}$ was determined. In bisulfite-modified DNA, unmethylated cytosine appeared as thymidine, whereas methylated cytosine remained unconverted. The percentage of methylated $\mathrm{CpG}$ at each site was calculated from all the PCR clones analyzed from each sample.

\section{Methylation-specific PCR (MSP)}

Methylation-specific PCR was performed according to published procedures. $^{18}$ Bisulfite-treated genomic DNA isolated from colon carcinoma cell lines $\mathrm{GC}_{3} / \mathrm{C} 1$, RKO and $\mathrm{Cac0} 2$ was analyzed. In the case of Caco2, DNA from cells previously treated with 5 -azadC $(1 \mu \mathrm{M})$ for $72 \mathrm{~h}$ was also analyzed. Genomic DNA was also extracted from paraffinembedded blocks of colon carcinoma specimens using the EX-WAX DNA extraction kit (Intergen) according to the instruction of the vendor. DNA was further purified (Wizard DNA clean-up kit, Promega) and subsequently bisulfite modified using the CpGenome DNA modification kit (Intergen). MSP primer sequences were designed that specifically recognize sequences containing either methylated $(\mathrm{CpG})$ or unmethylated (TpG) sites in the enhancer region of the first intron of the Fas gene. MSP reactions were performed on bisulfite-converted DNA. The following primers were used to discriminate between top-strand methylated and unmethylated $\mathrm{CpGs:}$

Methylated-F: 5'-AGTTTCGGCGTTTTTCGGAGATTATTGC

Methylated-R: $5^{\prime}$-CACCCGCGCCGAAACGAACC

Unmethylated-F: 5'-GGTAGTTTTGGTGTTTTTTGGAGATTATTGT

Unmethylated-R: 5'-CACCCACACCAAAACAAACCTTTAAC

PCR amplification of the selected region $(+630$ to +825$)$ in the first intron of the Fas gene to yield 184 and $187 \mathrm{bp}$ products with the methylated and unmethylated primer sets, respectively, was performed under the following conditions: initial denaturation at $94^{\circ}, 3 \mathrm{~min} ; 40$ cycles at $94^{\circ}, 1 \mathrm{~min} ; 64^{\circ}, 1 \mathrm{~min} ; 72^{\circ}, 2 \mathrm{~min}$; and a final extension of $72^{\circ}, 4 \mathrm{~min}$. The PCR products $(15 \mu \mathrm{l})$ were resolved on $2 \%$ agarose gels, stained with ethidium bromide and visualized by UV illumination.

\section{RT-PCR analysis of Fas mRNA expression}

Colon carcinoma cell lines were cultured in the presence or absence of $1 \mu \mathrm{M} 5$-azadC for $72 \mathrm{~h}$ in T75 flasks (Falcon), subsequently harvested and subjected to RNA extraction. Total RNA was isolated using the RNAqueous kit (Ambion, Austin, TX, USA). RNA (2 $\mu \mathrm{g})$ was reverse transcribed using cDNA Cycle Kit (Invitrogen, Carlsbad, CA, USA). Amplification of CDNA was performed using primers specific for Fas and $\beta$ actin (internal control). Primers specific for Fas receptor cDNA amplification were: (F) 5'-GACCCAGAATACCAAGTGCAGATGTA

(R) 5'-CTGTTTCAGGATTTAAGGTTGGAGATT

The primer pair specific for $\beta$-actin CDNA was:

(F) 5'-GTGGGGCGCCCCAGGCACCA

(R) 5'-CTCCTTAATGTCACGCACGATTTC

PCR amplification for Fas and $\beta$-actin mRNA was performed under the following conditions to yield 289 and 540 bp products, respectively: initial denaturation at $94^{\circ}, 3 \mathrm{~min} ; 25$ cycles at $94^{\circ}, 1 \mathrm{~min} ; 60^{\circ}, 2 \mathrm{~min}, ; 72^{\circ}, 2 \mathrm{~min}$; and a final extension of $72^{\circ}, 4 \mathrm{~min}$. The PCR products were resolved on $1.2 \%$ agarose gel, stained with ethidium bromide and visualized by UV illumination.

\section{Apoptosis assays}

$\mathrm{GC}_{3} / \mathrm{c} 1, \mathrm{RKO}$ and Caco2 cells were plated at a density of 200000 cells/ well in six-well plates. After overnight attachment, cells were cultured for $48 \mathrm{~h}$ in the presence or absence of $1 \mu \mathrm{M} 5$-azadC. Cells, both previously treated or untreated with 5-azadC, were further treated for $24 \mathrm{~h}$ with antiFas MoAb CH-11 (200 ng/ml; MBL International Corp.) or recombinant 
FasL ( $50 \mathrm{ng} / \mathrm{ml}$; Alexis) and M2 anti-Flag mAB ( $1 \mu \mathrm{g} / \mathrm{ml}$; Sigma). Both the floating cells and attached cells were pooled after trypsinization, fixed in $70 \%$ ethanol, and stored at $-20^{\circ} \mathrm{C}$ prior to the analysis. Apoptotic cells were detected as a sub-G1 fraction after propidium iodide staining and analysis using a Becton Dickinson FACScan. ${ }^{19,20}$

\section{Expression of cell surface Fas}

$\mathrm{GC}_{3} / \mathrm{c} 1$, RKO and Caco2 cells were plated at a density of 200000 cells/well in six-well plates. After overnight attachment, cells were cultured for $72 \mathrm{~h}$ in the presence or absence of 5 -azadC $(1 \mu \mathrm{M})$. Cell surface Fas expression was determined by flow cytometry using standard procedures. Throughout the whole study, the same lot (M044107) of phycoerythrin (PE)-labeled anti-Fas $\mathrm{mAB}$ (clone DX2; Pharmingen) was used. At each measurement, PE staining was calibrated using Quantibright (Becton Dickinson) beads. Nonviable cells were excluded using 7-aminoactinomycin D (7-AAD) staining. The mean fluorescence intensity values were converted to the number of $P E$ molecules/cell using Quantiquest software. Data were analyzed using the Student's $t$-test.

\section{Adenoviral delivery of wtp53}

Caco2 cells were plated as described earlier, and transduced either with a replication-incompetent wtp53 expressing adenoviral vector (Ad-wtp53; Genetic Therapy Inc.) at multiplicities of infection (moi) of 10, 30 and 100, or with an adenoviral vector control containing the basic vector backbone sequences (Ad-VC; moi 100). Cells were transduced with the adenoviral vectors either alone or in combination with 5-azadC $(1 \mu \mathrm{M})$ for $72 \mathrm{~h}$. Cells were subsequently harvested, and cell surface Fas expression determined by flow cytometric analysis as described. In order to asses Fas sensitivity, Caco2 cells were transduced with Ad-VC (moi 100) or Ad-wtp53 (moi 100) alone or in combination with 5 -azadC $(1 \mu \mathrm{M})$ for $48 \mathrm{~h}$ and subsequently incubated with or without $\mathrm{CH}-11(200 \mathrm{ng} / \mathrm{ml})$ for an additional $24 \mathrm{~h}$ in the presence or absence of cycloheximide (Sigma) $10 \mu \mathrm{M}$. Apoptosis was measured as described.

\section{Expression of Fas in vivo}

Sections from paraffin-embedded blocks of normal colon epithelium and 10 colon carcinomas were labeled with anti-Fas antibody (Dako, clone: APO-1, \# M3554) in 1:5 dilution after pronase digestion. Subsequently, peroxidase-labeled goat anti-mouse secondary antibody (Dako) was used for detection of APO-1 and developed using diaminobenzidine tetrahydrochloride (Sigma) following standard procedure. To assess morphology, hematoxylin stain was applied.

\section{Acknowledgments}

This research was supported by NIH Awards R01 CA 32613, R01 CA 14799, the Cancer Center Support (CORE) Grant CA 21765, and by the American Lebanese Syrian Associated Charities. I Petak was supported in part by a scholarship from the Fulbright Program, and by the 1st Institute of Pathology and Experimental Cancer Research, Semmelweiss University of Medicine, Budapest, Hungary.

The Hartwell Center for Bioinformatics and Biotechnology (St. Jude Children's Research Hospital) performed oligonucleotide synthesis and DNA sequence analysis.

\section{References}

1. Rouvier E, Luciani MF and Golstein P (1993) Fas involvement in $\mathrm{Ca}(2+)-$ independent T cell-mediated cytotoxicity. J. Exp. Med. 177: 195-200.

2. French LE, Hahne M, Viard I, Radlgruber G, Zanone R, Becker K, Muller C and Tschopp J (1996) Fas and Fas ligand in embryos and adult mice: I igand expression in several immune-privileged tissues and coexpression in adult tissues characterized by apoptotic cell turnover. J. Cell Biol. 133: 335-343.

3. Moller P, Koretz K, Leithauser F, Bruderlein S, Henne C, Quentmeier A and Krammer PH (1994) Expression of APO-1 (CD95), a member of the NGF/TNF receptor superfamily, in normal and neoplastic colon epithelium. Int. J. Cancer 57: 371-377.

4. Leithauser F, Dhein J, Mechtersheimer G, Koretz K, Bruderlein S, Henne C, Schmidt A, Debatin KM, Krammer PH and Moller P (1993) Constitutive and induced expression of APO-1, a new member of the nerve growth factor/tumor necrosis factor receptor superfamily, in normal and neoplastic cells. Lab. Invest. 69: 415-429.

5. Houghton JA, Harwood FG and Tillman DM (1997) Thymineless death in colon carcinoma cells is mediated via fas signaling. Proc. Natl. Acad. Sci. USA 94: 8144-8149.

6. Tillman DM, Petak I and Houghton JA (1999) A Fas-dependent component in 5-fluorouracil/leucovorin-induced cytotoxicity in colon carcinoma cells. Clin. Cancer Res. 5: 425-430.

7. Petak I, Tillman DM, Harwood FG, Mihalik R and Houghton JA (2000) Fasdependent and -independent mechanisms of cell death following DNA damage in human colon carcinoma cells. Cancer Res. 60: 2643-2650.

8. Eichhorst ST, Muerkoster S, Weigand MA and Krammer PH (2001) The chemotherapeutic drug 5-fluorouracil induces apoptosis in mouse thymocytes in vivo via activation of the CD95(APO-1/Fas) system. Cancer Res. 61: 243248.

9. Tillman DM, Harwood FG, Gibson AA and Houghton JA (1998) Expression of genes that regulate Fas signalling and Fas-mediated apoptosis in colon carcinoma cells. Cell Death Differ. 5: 450-457.

10. Vogelstein B, Fearon ER, Kern SE, Hamilton SR, Preisinger AC, Nakamura Y and White R (1989) Allelotype of colorectal carcinomas. Science 244: 207-211

11. Teitz $T$, Wei T, Valentine MB, Vanin EF, Grenet J, Valentine VA, Behm FG, Look AT, Lahti JM and Kidd VJ (2000) Caspase 8 is deleted or silenced preferentially in childhood neuroblastomas with amplification of MYCN. Nat. Med. 6: 529-535

12. Soengas MS, Capodieci P, Polsky D, Mora J, Esteller M, Opitz-Araya X, McCombie R, Herman JG, Gerald WL, Lazebnik YA, Cordon-Cardo C and Lowe SW (2001) Inactivation of the apoptosis effector Apaf-1 in malignant melanoma. Nature 409: 207-211

13. Behrmann I, Walczak H and Krammer PH (1994) Structure of the human APO1 gene. Eur. J. Immunol. 24: 3057-3062

14. Munsch D, Watanabe-Fukunaga R, Bourdon JC, Nagata S, May E, YonishRouach $E$ and Reisdorf P (2000) Human and mouse Fas (APO-1/CD95) death receptor genes each contain a p53-responsive element that is activated by p53 mutants unable to induce apoptosis. J. Biol. Chem. 275: 3867-3872

15. Petak I, Tillman DM and Houghton JA (2000) p53 dependence of Fas induction and acute apoptosis in response to 5 -fluorouracil-leucovorin in human colon carcinoma cell lines. Clin. Cancer Res. 6: 4432-4441

16. Butler LM, Dobrovic A, Bianco T and Cowled PA (2000) Promoter region methylation does not account for the frequent loss of expression of the Fas gene in colorectal carcinoma. Br. J. Cancer 82: 131-135

17. Danam RP, Qian XC, Howell SR and Brent TP (1999) Methylation of selected CpGs in the human 06-methylguanine-DNA methyltransferase promoter region as a marker of gene silencing. Mol. Carcinog. 24: 85-89

18. Herman JG, Graff JR, Myohanen S, Nelkin BD and Baylin SB (1996) Methylation-specific PCR: a novel PCR assay for methylation status of $\mathrm{CpG}$ islands. Proc. Natl. Acad. Sci. USA 93: 9821-9826

19. Gong J, Traganos F and Darzynkiewicz Z (1994) A selective procedure for DNA extraction from apoptotic cells applicable for gel electrophoresis and flow cytometry. Anal. Biochem. 218: 314-319

20. Mihalik R, Uher F, Pocsik EE, Berczi L, Benczur M and Kopper L (1996) Detection of drug-induced apoptosis by flow cytometry after alkaline extraction of ethanol fixed cells. Pathol. Oncol. Res. 2: 78-83. 